\title{
LA ACCIÓN TUTORIAL COMO ACOMPAÑAMIENTO EN EL ALUMNADO UNIVERSITARIO CON DISCAPACIDAD: HACIA UNA EDUCACIÓN INCLUSIVA
}

\author{
Gonzalo Lorenzo-Lledó \\ Universidad de Alicante.glledo@ua.es \\ Asunción Lledó Carreres \\ Alejandro Lorenzo Lledó \\ Graciela Arráez Vera
}

https://doi.org/10.17060/ijodaep.2017.n1.v4.1036

Fecha de Recepción: 9 Febrero 2017

Fecha de Admisión: 1 Abril 2017

\section{RESUMEN}

La situación actual de la discapacidad desde el marco normativo universitario nos depara un marco legislativo esperanzador por la apuesta hacia una educación inclusiva. La actual Ley Orgánica 4/2007, de 12 de abril (LOMLOU) establece en su Preámbulo el impulso de políticas activas para garantizar la igualdad de oportunidades a las personas con discapacidad. La disposición adicional vigésima cuarta, bajo el título de de la inclusión de las personas con discapacidad en las universidades presenta una serie de consideraciones previas para la elaboración de planes destinados a las personas con necesidades educativas especiales. Estas consideraciones irían al cumplimiento de preceptos como: igualdad de oportunidades, la no discriminación, provisión re decursos y apoyos, accesibilidad y una formación en la diversidad. Convertir los entornos educativos caracteritzats como discapacitantes en inclusivos es una tarea complia pero no imposible que en el momento actual no podemos dejar de resolver por la presencia de la discapacidad en el contexto universitario. El objetivo de este Trabajo ha sido diseñar un sistema de indicadores como herramienta de evaluación que determine variables personales y contextuales que pueden favorecer o dificultar la inclusión del estudiante universitario. Los resultados del sistema de indicadores desarrollado constatan como desde la acción tutorial se pueden llevar a cabo una serie de actuaciones de acompañamiento que favorecerían la inclusión del estudiante universitario con discapacidad. Concluimos con una apuesta por la acción tutorial que cubrirá en este alumnado un mayor seguimiento académico que redunde en la mejora de la calidad de su rendimiento y en una orientación académico-profesional para la atención a su proyecto personal.

Palabras Clave: acción tutorial, discapacidad, acompañamiento, indicadores, educación inclusiva. 


\title{
LA ACCIÓN TUTORIAL COMO ACOMPAÑAMIENTO EN EL ALUMNADO UNIVERSITARIO CON DISCAPACIDAD: HACIA UNA EDUCACIÓN INCLUSIVA
}

\begin{abstract}
The current situation of disability analyzed from the university regulatory framework give us a starting point to achieve an inclusive education. The current Organic Law 4/2007 of 12 April (LOMLOU) establishes in its Preamble the promotion of active policies to guarantee equal opportunities for people with disabilities. The twenty-fourth additional provision under the title of the inclusion of persons with disabilities in universities presents a series of preliminary considerations for the elaboration of plans for persons with special educational needs. These considerations would go to the fulfillment of precepts such as: equality of opportunities, non-discrimination, provision of resources and support, accessibility and diversity training. Turning the educational environments characterized as disabling in inclusive is a complicated but not impossible task that in the present moment we can not stop solving by the presence of the disability in the university context.The objective of this research has been to design a system of indicators as an evaluation tool that determines personal and contextual variables that can help or difficult the inclusion of university students. The results of the system of indicators developed show how from the tutorial action can be carried out a list of accompanying actions that can produce the inclusion of university students with disabilities. We conclude with a bet for the tutorial action as a tool that will cover in this students a greater academic follow-up. It will produce an improvement of the quality of their performance and in an academicprofessional orientation for the attention to their personal project.
\end{abstract}

Key Words: tutorial action, disability, accompaniment, indicators, inclusive education.

\section{ANTECEDENTES}

La sociedad del conocimiento que caracteriza el paradigma educativo actual demanda de cambios significativos e innovaciones que se traduzcan en actividades de acompañamiento y asesoramiento al estudiante universitario en su estancia en el contexto universitario. Estas innovaciones recientes en la Universidad del EEES aunque lleva incorporada algunos años, todavía carecen de una cultura arraigada y de una normalización plena en su implementación.

Desde hace ya algunos años se están implementando en las universidades españolas programas de acogida y acompañamiento al estudiante universitario en su proceso de adaptación al nuevo marco educativo en el que se demanda al alumnado un mayor protagonismo en sus aprendizajes (Lledó et al, 2014). El Informe Universidad Bricall (2000) ya indicaba como pieza fundamental en el nuevo escenario universitario, la figura del profesorado tutor del estudiante universitario. Ya se apuntan en dicho informe una serie de propuestas de actuación dirigidas al estudiante universitario para dar respuesta a las necesidades individuales de información, formación y de orientación en su diseño curricular y profesional.

Asimismo, la Ley Orgánica de Universidades (LOU) en su art. 46, establecía como derecho del estudiante universitario, el asesoramiento y asistencia por parte de profesores y tutores en el modo que se determine. Esta nueva tarea exige de nuevos elementos facilitadores de este proceso de construcción de los conocimientos tanto a nivel académico como personal y profesional. En este sentido se cubrirían aspectos como el asesoramiento previo al ingreso en la Universidad; el asesoramiento en la planificación y desarrollo de los estudios; los apoyos especiales, en casos de crisis o dificultades particulares, podrías ser cubiertos desde este tipo de actuaciones.

En este sentido la tutoría se convierte en una de las estrategias de apoyo al alumnado más eficaces puesto que constituye el primer nivel de concreción de orientación, la realizada por el profesor con su grupo de alumnos, bien sea de modo grupal o individualmente" (García Antelo, 2011, p.19). Por consiguiente, es inminente y necesaria la renovación en la forma que el profesorado transmite y evalúa el conocimiento de acuerdo con lo establecido en el marco europeo de educación 
superior. Es por lo que el profesorado universitario tiene que acometer un nuevo rol que le convierte en el referente del estudiante que llega a la universidad y que tendrá que realizar tareas de acompañamiento a lo largo del desarrollo de su itinerario profesional.

El profesorado universitario se convierte en el profesor de referencia del alumnado de nuevo ingreso en la universidad y de acompañamiento tutorial a lo largo de su proceso académico en el contexto universitario. De esta manera, la función tutorial se va a convertir en todo un conjunto de actividades, estrategias metodológicas orientadoras que desarrolla el profesorado tutor con sus alumnos tutorados con el objetivo de ayudarles a planificar su desarrollo académico, personal y profesional (Perandones y Lledó, 2009).

Los trabajos de Lledó et al (2008), han apostado por un nuevo perfil del docente universitario que cubra y amplíe hacia unas nuevas tareas: una información; universidad / estudiantes; un seguimiento académico que redunde en la mejora de su rendimiento; una intervención formativa desde la complementariedad curricular; y una orientación académico-profesional para la atención a su proyecto personal. Como apunta Rodríguez, (2004) se pueden establecer diferentes perspectivas para acometer esta función tutorial, desde una perspectiva centrada en la información que se realiza al estudiante sobre una asignatura en concreto (modelo académico); una perspectiva orientada más a las necesidades que se deben cubrir en cuanto a su desarrollo profesional (modelo de desarrollo profesional); y una tercera perspectiva que coincidiría con el actual planteamiento de la acción tutorial, en la que el asesoramiento y ayuda al estudiante amplia otros aspectos personales y profesionales que los estrictamente académicos.

Pero dentro de este escenario educativo universitario estaría incompleto sin tener en cuenta la diversidad existente hoy en día en las aulas universitarias. Nos referimos al estudiante con discapacidad. La nueva perspectiva de la Diversidad: la Educación Inclusiva está adentrándose aunque con lentitud en el contexto universitario, planteando nuevas direcciones en el marco de atención a la diversidad del alumnado universitario.

La Universidad, como institución educativa tiene que facilitar la incorporación de todo el alumnado, convirtiéndola en un entorno accesible y sin barreras en el aprendizaje, elaborando diseños curriculares donde se dé respuesta a todo el alumnado. El proceso de cambio en el que están inmersas las universidades españolas con el EEES no puede abandonar las actuaciones que vayan encaminadas a resolver las nuevas demandas que emanan de la diversidad existente en sus aulas (Lledó, Perandones, Sánchez, 2012).

Los datos reveladores procedentes del estudio realizado por la fundación Universia y el Comité Español de representatnes de personas con discapacidad (CERMI), sobre la situación actual y el nivel de inclusión de las personas con discapacidad en el sistema educativo universitario, curso 2011, 2012, en el que han paricipado 48 universidades españolas, indicaban que la presencia de este alumnado es de 12.75, correspondiendo al 69,69\% de la comunidad universitaria con discapacidad y al $1,1 \%$ de la comunidad universitaria general de las universidades participantes. En la actualidad, existen en España casi tres millones de personas con algún tipo de discapacidad igual o mayor al 33\%, según los datos del Instituto Nacional de Estadística (2015).

Adentrándonos en la legislación educativa universitaria, se establece en la Ley Orgánica 4/2007 que "la acción de la universidad no debe limitarse a la transmisión del saber; debe generar opinión, demostrar su compromiso con el progreso social y ser un ejemplo para su entorno. La igualdad entre hombres y mujeres, los valores superiores de nuestra convivencia, el apoyo permanente a las personas con necesidades especiales, el fomento del valor del diálogo, de la paz y de la cooperación entre los pueblos, son valores que la universidad debe cuidar de manera especial". Las pretensiones de la ley se alcanzarán partiendo de la propia práctica educativa del profesorado universitario 


\section{LA ACCIÓN TUTORIAL COMO ACOMPAÑAMIENTO EN EL ALUMNADO UNIVERSITARIO CON DISCAPACIDAD: HACIA UNA EDUCACIÓN INCLUSIVA}

que, desde sus diseños metodológicos, facilite una respuesta a la diversidad de capacidades, intereses y motivaciones de todo su alumnado, tal y como resalta el marco teórico de la educación inclusiva. Esta misma Ley establece seis principios básicos de actuación:

Igualdad de oportunidades, eliminando cualquier forma de discriminación y tomando medidas de acción positiva, que garanticen su participación plena y efectiva.

No discriminación en el acceso, ingreso, permanencia y ejercicio de sus títulos académicos.

Puesta en práctica de medidas de atención a las necesidades educativas especiales.

Accesibilidad a los servicios, recursos e instalaciones de todo el entorno universitario.

Desarrollo de los planes de estudio desde el respeto y la promoción de los Derechos Humanos y los principios de accesibilidad universal y diseño para todos.

Exención total de tasas y precios públicos en los estudios conducentes a la obtención de un título universitario.

Convertir los entornos educativos caracterizados como discapacitantes en inclusivos es una tarea compleja pero no imposible que en el momento actual no podemos dejar de resolver por la presencia de la discapacidad en el contexto universitario. Por consiguiente cuestionar que la discapacidad como elemento de diversidad está presente en la Universidad sería dirigir la mirada a otra realidad. Estamos abocados a abordar esta temática para ello se debe apostar apostar por un fuerte compromiso en el logro efectivo de los niveles de excelencia educativa deseados (Lledó, 2013).

En este sentido aspectos como el desconocimiento de la normativa actual, la no esxistencia de normativa que regule las medidas de atención a este alumnado, la existencia de barreras de acceso en las aulas, y como indican Moriña, Díez y Molina-Romo (2011) sobre una mayor información y formación al profesorado sobre atención a la diversidad, de la misma manera que hay que tener presente el recelo que suscitan las adaptaciones curriculares en la universidad, es necesario acometer estudios como el que se presentan para abordar un apoyo al estudiante con diversidad funcional asociada a discapacidad desde la acción tutorial.

\section{OBJETIVOS DE LA INVESTIGACIÓN}

Partiendo de la justificación teórica presentada, con el estudio que se presenta se plantea como objetivo general: diseñar un sistema de indicadores como herramienta de evaluación que determine variables personales y contextuales que pueden favorecer o dificultar la inclusión del estudiante universitario. Del mismo subyacen para su consecución una serie de objetivos más específicos como:

Analizar las necesidades y demandas del alumnado con diversidad funcional y hacerlas efectivas desde la acción tutorial.

Analizar la normativa actual sobre atención a la discapacidad en el contexto universitario.

Diseñar propuestas de actuación individualizada y tutoría entre iguales.

\section{MUESTRA DE PARTICIPANTES}

La muestra de participantes se ha conformado con 6 estudiantes, con discapacidad visual, (2), discapacidad auditiva (3) y TEA (1) que han constituido el estudio de casos del estudio realizado durante el curso académico 2016/2017, partiendo de trabajos anteriores.

\section{METODOLOGÍA E INSTRUMENTOS}

La metodología utilizada en función del objeto de estudio ha sido cualitativa centrada en el estudio de casos, como un proceso de indagación detallado, comprehensivo y sistemático y en profundidad del caso objeto de estudio (García Jiménez, 1991). Constituyen un campo privilegiado para conocer en profundidad los fenómenos educativos (Bartolomé, 1992, 24). Dicha metodología nos ha servido para analizar la situación de la discapacidad en los seis estudios de casos analizados y a 
partir de los mismos, diseñar el sistema de indicadores como instrumento de recogida de información sobre la atención hacia este alumnado y la implementación a través de la acción tutorial. El Instrumento utilizado ha sido un sistema de indicadores diseñado ad hoc a partir de las situaciones de los casos y la normativa legislativa en concreto de la propia universidad. Quedando conformado con los siguientes indicadores (tabla 1)

Tabla 1.

Sistema de indicadores de evaluación para el estudio de casos

\section{Indicadores}

1. Conocimiento de la discapacidad en el acceso a la Universidad.

2. Existencia de legislación educativa reguladora de medidas de atención a la discapacidad.

3. Conocimiento de existencia de centro de apoyo al estudiante con discapacidad.

4. Solicitud y tramitación de medidas de adaptaciones curriculares y de acceso para atender a las necesidades de cada discapacidad.

5. Conocimiento por parte del profesorado de las medidas reguladoras existentes para atender a la discapacidad.

6. Aulas y materiales accesibles.

7. Actitud de disponibilidad e implicación del profesorado hacia las medidas propuestas.

8. Necesidad de una mayor formación del profesorado en el conocimiento de la DV.

9. Necesidad de una mayor formación del profesorado en el conocimiento de la DA.

10. Necesidad de una mayor formación del profesorado en el conocimiento de los trastornos del espectro autista (autismo y síndrome de Asperger)

11. Apoyo y colaboración por parte de los compañeros y compañeras del grupo.

12. Conocimiento de la existencia del programa de acción tutorial (PAT) de la universidad.

\section{RESULTADOS}

Los primeros resultados del estudio que está pendiente por finalizar, han dejado constancia de los siguientes resultados (tabla 2): 
Tabla 2.

Presencia o ausencia de indicadores en el estudio de casos de la muestra

\begin{tabular}{|c|c|}
\hline Indicadores & Presencia/ausencia \\
\hline $\begin{array}{l}\text { 1. Conocimiento de la discapacidad en el acceso } \\
\text { a la Universidad. }\end{array}$ & Ausencia de coordinación. \\
\hline $\begin{array}{l}\text { 2. Existencia de legislación educativa reguladora } \\
\text { de medidas de atención a la discapacidad y } \\
\text { contrato de aprendizaje. }\end{array}$ & $\begin{array}{l}\text { Presencia pero con tramitación lenta para realizar el } \\
\text { contrato de aprendizaje. }\end{array}$ \\
\hline $\begin{array}{l}\text { 3. Conocimiento de existencia de centro de } \\
\text { apoyo al estudiante con discapacidad. }\end{array}$ & Presencia y apoyo regular muy útil. \\
\hline $\begin{array}{l}\text { 4. Solicitud y tramitación de medidas de } \\
\text { adaptaciones curriculares y de acceso para } \\
\text { atender a las necesidades de cada } \\
\text { discapacidad. }\end{array}$ & $\begin{array}{l}\text { Presencia pero proceso de tramitación lento y } \\
\text { burocrático. }\end{array}$ \\
\hline $\begin{array}{l}\text { 5. Conocimiento por parte del profesorado de las } \\
\text { medidas reguladoras existentes para atender a } \\
\text { la discapacidad. }\end{array}$ & $\begin{array}{l}\text { Falta de conocimiento en todas las discapacidades } \\
\text { aunque diversidad en la implicación del profesorado. } \\
\text { Más complejo en los casos de TEA. Dependiendo } \\
\text { también de las asignaturas. }\end{array}$ \\
\hline 6. Aulas y materiales accesibles. & $\begin{array}{l}\text { Presencia de accesibilidad aunque ausencia de } \\
\text { apuntes adaptados (letra) y recursos TIC (FM, } \\
\text { ordenadores). }\end{array}$ \\
\hline $\begin{array}{l}\text { 7. Actitud de disponibilidad e implicación del } \\
\text { profesorado hacia las medidas propuestas. }\end{array}$ & $\begin{array}{l}\text { Presencia reducida de aplicación de las medidas } \\
\text { establecidas. }\end{array}$ \\
\hline $\begin{array}{l}\text { 8. Necesidad de una mayor formación del } \\
\text { profesorado en el conocimiento de la DV. }\end{array}$ & $\begin{array}{l}\text { Ausencia generalizada de formación en discapacidad. } \\
\text { Es el alumnado el que tiene que informar al } \\
\text { profesorado aunque la coordinadora de discapacidad } \\
\text { de la Facultad también les informa. }\end{array}$ \\
\hline $\begin{array}{l}\text { 9. Necesidad de una mayor formación del } \\
\text { profesorado en el conocimiento de la DA. }\end{array}$ & Ausencia generalizada de formación en discapacidad. \\
\hline $\begin{array}{l}\text { 10. Necesidad de una mayor formación del } \\
\text { profesorado en el conocimiento de los } \\
\text { trastornos del espectro autista (autismo y } \\
\text { síndrome de Asperger) }\end{array}$ & Ausencia mayor del conocimiento de los TEA. \\
\hline $\begin{array}{l}\text { 11. Apoyo y colaboración por parte de los } \\
\text { compañeros y compañeras del grupo. }\end{array}$ & $\begin{array}{l}\text { Presencia de disponibilidad e implicación de la } \\
\text { tutoría entre iguales. }\end{array}$ \\
\hline $\begin{array}{l}\text { 12. Conocimiento de la existencia del programa } \\
\text { de acción tutorial (PAT) de la universidad. }\end{array}$ & $\begin{array}{l}\text { Asistencia por recomendación al PAT y satisfacción } \\
\text { total por parte del alumnado. }\end{array}$ \\
\hline
\end{tabular}

\section{DISCUSIÓNES}

Trabajos anteriores (Lledó, 2015 y 2016) como los actuales, muestran que hay una necesidad significativa de formación sobre la atención a este alumnado así como el conocimiento de sus necesidades educativas. Siendo este uno de los retos pendientes que tienen que protagonizar las universidades: por una parte, la necesidad de establecer unas orientaciones previas al alumnado que va a acceder al contexto universitario por parte de los especialistas de apoyo psicopedagógico de 
los centros no universitarios, como por otra, la normalización y puesta en práctica de las adaptaciones curriculares no significativas. Entendidas como la estrategia educativa que con modificaciones 0 ajustes no significativos del currículum ordinario que permiten al alumnado el acceso y promoción al currículo ordinario sin perjuicio de la adquisición de la competencias profesionales y los contenidos académicos que establecen los títulos universitarios y que habilitan para el ejercicio profesional, así como las Adaptaciones de apoyo en el aula como modificaciones o provisión de recursos espaciales, materiales, personales, de comunicación y didácticos para facilitar el acceso al currículum ordinario (Reglamento de adaptación curricular de la Universidad de Alicante, 2015). Sin olvidar las modificaciones y adaptaciones en las pruebas de evaluación programadas en cada asignatura que suponen adaptaciones curriculares en las pruebas de evaluación.

\section{CONCLUSIONES}

Los presupuestos teórico-prácticos de la Educación Inclusiva pueden ser una respuesta de mejora hacia la consecución de una excelencia educativa y accesibilidad de todos en el contexto universitario.

En función de los objetivos programados, se ha podido con la metodología utilizada, analizar de manera individualizada los distintos estudios de caso que conforman la muestra de participantes y a partir del sistema de indicadores diseñado, abordar la presencia 0 ausencia de los mismos y las actuaciones que de ellos se derivan y a partir de ello, orientar una respuesta educativa en función de cada discapacidad. Concluyendo con:

La falta de formación y cultura educativa del profesorado universitario para realizar propuestas de trabajo adaptadas a la discapacidad.

La gran mayoría del profesorado mira con recelo los cambios curriculares considerados como trabajo añadido y en algunos casos por desconocimiento.

La pertenencia a grupos tutoriales por parte de estudiantes con discapacidad ha hecho posible poder expresar sus dificultades y ser más conocidas y comprendidas tanto por el profesor tutor como por el propio alumnado.

Los resultados de las actuaciones implementadas indican que el asesoramiento y acompañamiento que ofrece la acción tutorial ha supuesto una mejora a favor de un mayor conocimiento del alumnado tutorado así como la necesidad de incorporar la variable diversidad funcional en las estrategias metodológicas del profesorado.

La acción tutorial y la tutoría entre iguales han puesto de manifiesto resultados muy satisfactorios entre el alumnado participante, reconociendo y valorando con la normalidad las dificultades que presentan y que son más comprendidas por sus compañeros y compañeras y por el propio profesorado.

Una vez concluido el curso académico 2016/2017 se podrán obtener todos los resultados completos y que serán objeto de posibles informes para conocimiento de la comunidad educativa.

\section{REFERENCIAS BIBLIOGRÁFICAS}

Álvarez Pérez, P. (2006). La tutoría y la orientación universitaria en la nueva coyuntura de la enseñanza superior: el Programa Velero. Revista Contextos educativos, 8-9, pp-281-293.

Bartolomé, M. (1992). Investigación cualitativa: ¿Comprender o transformar? Revista de Investigación educativa, 20 (2), 7-36.

BOUA de 28 de julio de 2015. Reglamento de adaptación curricular de la Universidad de Alicante.

Bricall, J.M. (2000). Informe Universidad 2000. Madrid: CRUE.

Fundación Universia (2014). Universidad y discapacidad. II estudio sobre el grado de inclusión del sistema universitario español respecto de la realidad de la discapacidad. Madrid: Servicio de 


\section{LA ACCIÓN TUTORIAL COMO ACOMPAÑAMIENTO EN EL ALUMNADO UNIVERSITARIO CON DISCAPACIDAD: HACIA UNA EDUCACIÓN INCLUSIVA}

información sobre la discapacidad. Recuperado de http://sid.usal.es/bdocus/discapacidad/26780/8-4-1/ii-estudio-sobre-el-grado-de-inclusion-del-sistema-universitario-espanol-respecto-de-la-realidad-de-la-discapacidad.aspx.

García Antelo, B. (2011). La tutoría universitaria: percepción de alumnado y profesorado. Universidade de Santiago de Compostela: Servicio de Publicaciones e Intercambio Científifico.

García Jiménez, E. (1991). Una teoría práctica sobre la evaluación. Estudio etnográfico. Sevilla: MIDO.

Instituto Nacional de Estadística (2015). Base estatal de datos de personas con valoración de grado de discapacidad (Informe a 31/12/2014) Recuperado de http://imserso.es/InterPresent2/groups /imserso/documents/binario/bdepcd_2014.pdf

Ley Orgánica 6/2001, de 21 de diciembre, de Universidades (LOU). (BOE núm. 307, de 24/12/2001).

Lledó, A. (coord.), Baile, E., Cámara, H., Delgado, B., Fernández, C., Gilar, R., González, C., Gonzálvez, C., Hernández, M.J., Lorenzo, G., Llorens, R., Merma, G., Perandones, T., Rovira, J. (2014). Un trabajo colaborativo y de acompañamiento tutorial: el Plan de Acción Tutorial en la Facultad de Educación, En Álvarez, Tortosa y Pellín. Investigación y Propuestas Innovadoras de Redes UA para la Mejora Docente. Ediciones Universidad de Alicante.

Lledó Carreres, A. (2015). La inclusión educativa de la discapacidad en la universidad; una cuestión pendiente para seguir avanzando en Educación Superior. En Lucía Herrera (coord.) Retos y desafíos de la Educación Superior desde la perspectiva del profesorado universitario. Madrid: Síntesis.

Lledó, A., Lorenzo, G., Gonzálvez, C., Vicent, J., Martínez, M., Veas, A., Soler, R., Aparicio, P., Hernández, M.J., Vogel, E., y Delgado, B. (2016). La acción tutorial para el alumnado con diversidad funcional en la Universidad. En Tortosa, Grau y Álvarez. Investigación, innovación y enseñanza universitaria: enfoques pluridisciplinares. Universidad de Alicante: ICE de la UA.

Lledó, A., Perandones, TM., Sánchez, J.F. (2012). La diversidad en la Universidad: una cuestión pendiente. International Journal of Developmental and Educational Psychology, INFAD Revista de Psicología, 1 (3), 147-157.

Lledó, A; Álvarez, JD; Grau, S; González, C; Roig, R; Lorenzo, G; Tortosa, MT. (2008). Academia learning and achievement The mentoring program in the university as a measure of quality the learning students's process: A proposal for action at the university of Alicante. XXIX. Congress of Psychology. Berlin. Alemania.

Moriña, Díez, A. y Molina- Romo, V. (2011). La universidad a análisis: las voces del alumnado con discapacidad. Revista de Enseñanza Universitaria, 37, 23-35.

Perandones, T.M. y Lledó, A. (2009) La función del profesorado universitario como tutor. Experiencias en el Programa de Acción Tutorial en la Universidad de Alicante. En Roig, R.; Blasco, J.; Gilar, R.; Lledó, A.; Mañas, C.; (eds.). Investigar desde un contexto educativo innovador. Alcoy: Marfil.

Rodríguez, S (2004). Manual de tutoría universitaria. Barcelona: Octaedro/ICE-UB. 